Global Journal of Pure and Applied Mathematics

ISSN 0973-1768 Volume 5, Number 1 (2009), pp. 89-94

(C) Research India Publications

https://dx.doi.org/10.37622/GJPAM/5.1.2009.89-94

\title{
On semiprime, Prime and Strongly Prime $*$-bi-ideals in Semigroup with Involution
}

\author{
Moin A. Ansari, M. Rais Khan and J.P. Kaushik \\ Department of Mathematics, Faculty of Natural Sciences \\ Jamia Millia Islamia (Central University) \\ New Delhi-110025
}

\begin{abstract}
In [1] Ahsan and Liu has characterized the prime (semiprime) ideals and right ideals for a semigroup. Furthermore G. Szasz[5] has considered semigroups in which ideals are prime. He has also shown that the ideals of a semigroup P are prime if and only if $\mathrm{P}$ is intra regular. In this paper we have introduce the concept of semiprime, prime and strongly prime *-bi-ideals in semigroups with involution. Also we have given characterizations for involution semigroups whose bi-ideals are having all the above properties. Furthermore we have investigated their structural properties by attaching involution on their corresponding ground semigroups. For every involution semigroup $\mathrm{S}$ we denote *-bi-ideal for bi-ideals in involution semigroup.
\end{abstract}

AMS Mathematics Subject Classification (2000): 20M10

Key words and phrases: Involution semigroups, Prime, Semiprime, Strongly prime *-bi-ideals.

\section{Introduction}

In [1] Ahsan and Liu has characterized the prime (semiprime) ideals and right ideals for a semigroup. Furthermore G. Szasz [5] has considered semigroups in which ideals are prime. All definitions and fundamental concepts concerning semigroups and their involution properties can be found in [2] and [4]. In this section we have given related definitions based on prime, semiprime and strongly prime *-bi-ideal in involution semigroups. Also we have given some examples. Throughout this paper we have assumed $\mathrm{S}$ with a zero element. For different notations and terminologies the reader is referred to [2]. For every involution semigroup $\mathrm{S}$ we denote *-bi-ideal for bi-ideals in 
semigroup. Let $B$ be a *-bi-ideal then $B(a)$ denote *-bi-ideal generated by an element a.

Definition 1.1: [6] A *-semigroup is a set $\mathrm{S}$ equipped with a binary operation : and a unary operation $* S \rightarrow S$ satisfying the following three axioms:

(i) $(x, y), z=x,(y, z)$

(ii) $\left(a^{*}\right)^{n}=a^{*+*}=a$

(iii) $\quad(a b)^{*}=b^{*} a^{*} \forall a_{y} b \in S^{\prime}$.

Such a unary operation $*$ is sometimes called an involution, and $(\mathrm{S}, \mathrm{e}, *)$ is

sometimes called an involution semigroup.

Let A be a non-empty subset of a *-semigroup $(S, *)$ ) then $\mathrm{A}$ is said to be a *subsemigroup of $S$ if $a b \in A$ for all $a, b \in A$. Where $*$ is taken as inverse of an element. Somewhere $*$ is taken as transpose in matrix sense and somewhere it is taken as inverse in general set theoretical sense. $A$ *-subsemigroup $B$ of an involution semigroup $S$ is called a $*$-bi-ideal of $S$ if $B S B \subseteq \mathrm{B}$ with the condition $B^{n} \leq B$ and $B^{n}=\left\{b^{*} \in S: b \in B\right\}[3]$. It is well known that intersection of any two *-bi-ideal of an involution semigroup $S$ is a *-bi-ideal and in more general way intersection of a finite number of *-bi-ideal is again a *-bi-ideal. Also product of any two *-bi-ideal is a *-biideal of $\mathrm{S}$.

Definition1.2: Let $B$ be a $*$-bi-ideal of $S$ then $B$ is said to be a prime *-bi-ideal of $S$ if $B_{1} B_{2} \subseteq B$ implies that $B_{1} \subseteq B$ or $B_{2} \subseteq B$, where $B_{1}, B_{2}$ both are *-bi-ideal of $\mathrm{S}$. Equivalently, $S-B$ is a *-semigroup. Analogously we can say that a *-bi-ideal B is completely prime if for any two $b_{1}, b_{2} \in B$ implies that $b_{1} \in B$ or $b_{2} \in B$ where $b_{1}, b_{2}$ being the elements of $\mathrm{S}$. A *-bi-ideal $\mathrm{B}$ which is completely prime is prime.

Example1.3: An involution semigroup $S$ itself is always a prime *-bi-ideal of $S$.

Examples1.4: Suppose that $S=[0,1[$ be an involution semigroup under the * operation taken as inverse of numbers between 0 and 1 with respect to multiplication (Excluding 1) where $a \in\left[0,1\left[\right.\right.$. Considering an element $\frac{1}{2}$ with any other subset $C_{1}$ such that,

$S=\epsilon_{1} \cup\left\{\frac{1}{2}\right\}$. Now suppose o denote commutative multiplication in such a way that:

$a \circ b=\left\{a b\right.$, if $\left.a \in C_{1}, b \in C_{1}\right\}$

$a \circ b=\left\{0\right.$, if $\left.a \in C_{1}, b=\frac{1}{2}\right\}$

$a \circ b=\left\{\frac{1}{4}\right.$, if $\left.a=\frac{1}{2}, b=\frac{1}{2}\right\}$ 
Then the set $B=\left\{0, \frac{1}{2}\right\}$ is a prime *-bi-ideal of $S$.

Example 1.5: Suppose that $S=M_{2 \times 2}\left(\mathbb{Z}_{4}\right)$ be the $2 \times 2$ matrix *-semigroup over the semigroup $\mathbb{Z}_{4}$. Then $P(B)=\left\{\left(\begin{array}{ll}a_{11} & a_{12} \\ a_{18} & a_{14}\end{array}\right) \mid a_{i 1} \in Z_{2}\right\}$.

Definition 1.5: Let $B$ be a $*$-bi-ideal of $S$ then $B$ is said to be a semiprime *-bi-ideal of $\mathrm{S}$ if $\forall B_{1} \in B, B_{1}^{2} \subseteq B \Rightarrow B_{1} \subseteq B$.

Definition 1.6: Let B be a *-bi-ideal then B is said to be a strongly prime *-bi-ideal of $\mathrm{S}$ if for any two *-bi-ideals $B_{1}$ and $B_{2}$ of $\mathrm{S}$ we have,

$$
B_{1} B_{2} \cap B_{2} B_{1} \subseteq B \Rightarrow B_{1} \subseteq B \text { or } B_{2} \subseteq B \text {. }
$$

Remarks: Every strongly prime *-bi-ideal of an involution semigroup $\mathrm{S}$ is a prime *bi-ideal and every prime *-bi-ideal is a semiprime *-bi-ideal. A prime *-bi-ideal is not necessarily strongly prime and a semiprime *-bi-ideal is not necessarily prime.

Lemma 1.7: Now suppose that $S t P(B)$ denote strongly prime *-bi-ideal, $S_{e} P(B)$ denote semiprime *-bi-ideal and $\mathrm{P}(\mathrm{B})$ denote prime *-bi-ideal of $\mathrm{S}$. Then $\operatorname{StP}(B) \subseteq P(B) \subseteq \operatorname{SeP}(B)$.

Proof: Suppose that $B$ is a strongly prime *-bi-ideal i.e. suppose $B \in \mathrm{StP}(\mathrm{B})$. Our aim is to show that $B \in P(B)$. To that end let $C D \subseteq B$. Since $B$ is strongly prime so $C \subseteq B$ or $D \subseteq B$. So $B$ is prime. Which implies that $\operatorname{StP}(B) \subseteq \mathrm{P}(B)$. Now suppose that $C^{2} \subseteq B$. Then clearly, $C \subset \subseteq B$ and so $C \subseteq B$. Hence $B$ is semiprime. Finally, $\mathrm{P}(\mathrm{B}) \mathrm{I}) \mathrm{S} \mathbb{P}(\mathrm{E})$. It follows from the definition itself.

Example 1.8: Consider the semigroup $S=\{0,1,2\}$ defined in tabular form as follows:

\begin{tabular}{l|l|l|l}
. & 0 & 1 & 2 \\
\hline 0 & 0 & 0 & 0 \\
\hline 1 & 0 & 1 & 1 \\
\hline 2 & 0 & 1 & 2
\end{tabular}

Here *-bi-ideals of $S$ are $\{0\},\{0,1\},\{0,2\}$ and $\{0,1,2\}$.

Therefore, all *-bi-ideals are prime *-bi-ideals and hence semiprime *-bi-ideals. However, the prime $*$-bi-ideals $\{0\}$ is not strongly $\{0\}$ prime $*$-bi-ideals because

$\{0,1\}\{0,2\} \cap\{0,2\}\{0,1\}=\{0,1\} \cap\{0,2\}=\{0\} \subseteq\{0\}$.

Example 1.9: All *-maximal ideals of involution semigroup $S$ are strongly prime *bi-ideals. 
Lemma1.10: Let $B_{1}$ and $B_{2}$ be any two prime *-bi-ideals of an involution semigroup $\mathrm{S}$ then $B_{1} \cap B_{2}$ is a prime *-bi-ideals then either $B_{1} \subseteq B_{2}$ or $B_{2} \subseteq B_{1}$.

Proof: It is clear that $B_{1} B_{2} \subseteq B_{1} \cap B_{2}$. Because $B_{1} \cap B_{2}$ is prime *-bi-ideals (By assumption) of $\mathrm{S}$ so either $B_{1} \subseteq B_{1} \cap B_{2}$ or $B_{2} \subseteq B_{1} \cap B_{2}$ and hence either $B_{1} \subseteq B_{2}$ or $B_{2} \subseteq B_{1}$.

Theorem 1.11: For an involution semigroup $S$ the following assertions are true:

(i) $B^{2}=B$ for every $*$-bi-ideal $B$ of $\mathrm{S}$.

(ii) $B_{1} \cap B_{2}=B_{1} B_{2} \cap B_{2} B_{1}$ for all *-bi-ideal $B_{1}$ and $B_{2}$ of $\mathrm{S}$.

(iii) Each *-bi-ideal of $\mathrm{S}$ is semiprime.

Proof: $(t) \Rightarrow(t i)$ Let $B_{1}$ and $B_{2}$ be any two -bi-ideals of $S$. Then by our hypothesis, $B_{1} \cap B_{2}=\left(B_{1} \cap B_{2}\right)^{2}$

$$
\begin{aligned}
& =\left(B_{1} \cap B_{2}\right)\left(B_{1} \cap B_{2}\right) \\
& =B_{1}
\end{aligned}
$$

In the similar way,

We have $B_{1} \cap B_{2} \subseteq B_{2} B_{1}$

$$
B_{1} \cap B_{2} \subseteq B_{1} B_{2} \cap B_{2} B_{1}
$$

Here it is clear that $B_{1} B_{2}$ and $B_{2} B_{1}$ both are *-bi-ideals being the product of *-biideals. Moreover $B_{1} B_{2} \cap B_{2} B_{1}$ is also a *-bi-ideals. And hence,

$$
\begin{aligned}
B_{1} B_{2} \cap B_{2} B_{1} & =\left(B_{1} B_{2} \cap B_{2} B_{1}\right)\left(B_{1} B_{2} \cap B_{2} B_{1}\right) \\
& \subseteq B_{1} B_{2} B_{2} B_{1} \\
& \subseteq B_{1} S B_{1} \\
& \cong B_{1}
\end{aligned}
$$

In the same way we can have,

$B_{1} B_{2} \cap B_{2} B_{1} \subseteq B_{2}$

And hence we can say that, $B_{1} B_{2} \cap B_{2} E_{1} \subseteq B_{1} \cap B_{2}$

Therefore by above two conditions we have, $B_{1} B_{2} \cap B_{2} B_{1}=B_{1} \cap B_{2}$

$(i i i) \Rightarrow(i v)$. For this we let that $E_{1}$ and $E_{2}$ be any two $*$-bi-ideals of $S$ such that $B_{1}^{2} \subseteq B$. Which implies by our supposition that,

$B_{1}=B_{1} \cap B_{1}=B_{1} B_{1} \cap B_{1} B_{1}=B_{1}^{*}$

So,

$B_{1} \subseteq B$.

Hence every *-bi-ideal is semiprime.

Lemma 1.12: The intersection of family of prime *-bi-ideals of a commutative involution semigroup is a semiprime *-bi-ideals. 
Proof: We'll show this result for two prime *-bi-ideals and then generalize for family of prime *-bi-ideals. Suppose that $B_{1}$ and $B_{2}$ be any two prime *-bi-ideals of an involution semigroup $S$. Now for any $x \in S, x^{2} \in B_{1} \cap B_{2} \Rightarrow x^{2} \in B_{1}$ and $x^{2} \in B_{2}$. Since $\bar{B}_{1}$ is a prime *-bi-ideal of $\mathrm{S}$. Therefore, $x^{2}=x: x \in B_{1} \Rightarrow x \in B_{1}$. In the same way, $x^{2}=x_{1} x \in B_{2} \Rightarrow x \in B_{2}$. Hence $x \in E_{1} \cap B_{2}$.

Theorem1.13: Let $\mathrm{S}$ be a semigroup with involution then the following assertion are true:

(i) $(A)^{2}=A$ for every *-bi-ideal $\mathrm{A}$ of $\mathrm{S}$.

(ii) $A \cap C=A C$ for every $*$-bi-ideals $A$ and $C$ of $\mathrm{S}$.

(iii) $B(a) \cap B(b)=B(a) B(b)$ for all $a_{z} b \in S$.

(iv) $B(a)=(B(a))^{2}$ for all $a \in S$.

(v) $a \in(B a B a B)$ for all $a \in S$.

Proof: (i) is followed by (ii): Consider $A$ and $C$ be -bi-ideal of $S$. Then $A C \subseteq A S \subseteq A$ in other way $A C \subseteq S C \subseteq C$ i.e; $A C \subseteq A \cap C$. As $A \cap C$ is a *-bi-ideal of S[give reference of intersection of bi-ideal in again a bi-ideal] therefore we have,

$A \cap C=(A \cap C)^{2}=(A \cap C)(A \cap C) \subseteq A C$.

(tt) is followed by $(t t)$ : For this we consider that $a_{n} b \in S$. Since $B(a), B(b)$ are *-bi-ideal of S. Hence,

$B(a) \cap B(b)=B(a) B(b)$ by (ii).

$(t i i)$ is followed by (iv): Straight forward.

(tv) is followed by (v): Let $a \in S$. Here $B(a)=(B(a))^{2}$ thus we have,

$(B(a))^{2}=(B(a))^{2} B(a) \subseteq(B(a))^{3} \subseteq(B(a))^{3} B(a) \subseteq(B(a))^{4}$.

$\Rightarrow(B(a))^{4} \subseteq(B(a))^{3}$.

Therefore we have,

$$
\begin{aligned}
B(a) & =(B(a))^{2} \subseteq(B(a))^{3} \subseteq(B(a))^{4} \\
& =(B(a))^{4} \subseteq(B(a))^{3}=(B(a))^{3} \\
& \subseteq(S B(a)) \subseteq(B(a))=(B(a)) \\
& \Rightarrow B(a)=(B(a))^{3} .
\end{aligned}
$$

In the similar way we can have,

$$
\begin{aligned}
(B(a))^{2}=((a \cup S a \cup a s \cup S a s))^{2} & \\
& \subseteq((a \cup S a \cup a S \cup S a s))^{2}(a \cup S a \cup a s \cup S a S) \\
& \subseteq(S a \cup S a S)(a \cup S a \cup a S \cup S a S)
\end{aligned}
$$




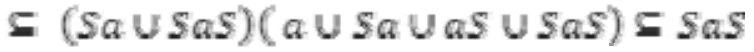

$$
\begin{aligned}
& \Rightarrow(B(a))^{4}=(S a S)(a \cup S a \cup a S \cup S a S) \\
& \text { - (SaSaUSaS } \left.a \cup S a S a S \cup S a S^{2} a S\right) \\
& =(S a S a U S a S a S) \\
& \Rightarrow(B(a))^{2}=(S a S a \cup S a S a S)(a \cup S a \cup a S \cup S a S) \\
& \text { G }(\operatorname{SaS} a S) \text {. }
\end{aligned}
$$

And hence finally we have,

$a \in B(a)=(B(a))^{2} \subseteq($ SaSaS $)=$ SaSaS.

(v) is followed by $(t)$ : For this we suppose that $B_{1}$ be $*$-bi-ideal of S. Next suppose that $x \in B_{1}^{2}$. Since $B_{1}$ is a $*$-bi-ideal of $S$. Therefore, $a b \in B_{1}$,

Further let $x \in B_{1}$ then by (v), $x \in(B x B x B)$. Also since,

$(t x) n \in\left(S B_{1}\right) S \subseteq B_{1} S \subseteq B_{1}$, also $x k \in B_{1} S \subseteq B_{1}$. Therefore we have, $x \in B_{1}^{2}$ for some $t_{z} n_{\nu} k \in S$.

Theorem 1.14: Let $S$ denote semigroup with involution and $B$ be *-bi-ideal of $S$ then $\mathrm{B}$ is strongly prime *-bi-ideal of $\mathrm{S}$ if and only if one of the five equivalent conditions of lemma 1.9 holds in $\mathrm{S}$.

\section{References}

[1] J. Ahsan and Zhongkui Liu: Prime and semiprime acts over monoids with zero, Math. J. Ibaraki Univ. 33, 9-15 (2001)

[2] J. M. Howie, Fundamentals of semigroup theory, Oxford University Press, London, 1995.

[3] K.I. Beidar, L.MÁRKI, R.MLITZ and WIEGANDT, Primitive inv olution rings, Acta Math. Hungar. 109(4) (2005), 357-368

[4] Max-Albert Knus, Alexander Merkurjev, Markus Rost and Jean-Pierre Tigno, AMS Colloquium Publications, Vol. 44, 1998.

[5] Szasz, G., Eine Charakteristic der Primeidealha-lbgroupen, Publ. Math Debrecen 17 (1970), 209-213)

[6] T.E. Nordahl and H.E. Scheiblich, Regular *-semigroups, semigroup forum vol. 16(1978) 369-377 